\title{
ARBITRAJE Y MÚLTIPLES CONTRATOS
}

ARBITRATION AND MULTIPLE CONTRACTS

\author{
Fernando Cantuarias Salaverry ${ }^{\star}$ \\ Universidad del Pacífico \\ José Luis Repetto Deville \\ Miranda \& Amado
}

\begin{abstract}
The globalized world demands increasingly complex contractual operations. In that context, businesses have implemented multiple related contracts with the aim of achieving a common goal.

In this paper, the authors analyze the complex situation of the arbitration regarding multiple contracts. Through the review of relevant case-law, the various solutions that characterize arbitration between multiple contracts are described. Finally, the authors address the overlap of non-signatory parties in the context of multiple contracts.
\end{abstract}

KEYWORDS: Arbitration; Multi-Contract; Group of Contracts; Arbitration Agreement; Complex Arbitrations.
El mundo globalizado exige operaciones contractuales cada vez más complejas. En ese contexto, los negcios han implementado multiples contratos conexos con el objetivo de conseguir un fin común.

En el presente artículo, los autores analizan la compleja situación del arbitraje en el contexto de múltiples contratos. A través de la revisión de la jurisprudencia relevante, se describen las diversas soluciones que caracterizan un arbitraje frente a múltiples contratos. Finalmente, los autores abordan la superposición de partes no signatarias en el contexto de múltiples contratos.

Palabras Clave: Arbitraje; Multi-Contratos; Grupo de Contratos; Convenio Arbitral; Arbitrajes Complejos.

* Abogado. Master en Derecho (LL.M.) por la Universidad de Yale. Decano de la Facultad de Derecho de la Universidad del Pacífico. Profesor de Arbitraje Comercial y Arbitraje de las Inversiones. Árbitro nacional e internacional. Miembro del Board of Reporters del Institute of Transnacional Arbitration (ITA), del Grupo Latinoamericano de Arbitraje de la Cámara de Comercio Internacional (CCI), de la Asociación Americana de Derecho Internacional Privado (ASADIP), de la Association for International Arbitration (AIA), de la Asociación Latinoamericana de Arbitraje y del Comité Argentino de Arbitraje Nacional y Transnacional (CARAT). Miembro de la lista de árbitros de los principales Centros de Arbitraje del Perú y del Energy Arbitrators' list. Es co.autor de la Ley de Arbitraje peruana de 2008. Contacto: cantuarias_f@up.edu.pe.

** Abogado. Ha sido presidente del Consejo Directivo de la Asociación DERUP Editores - Forseti, conformada por alumnos de la Facultad de Derecho de la Universidad del Pacífico. Es autor de publicaciones de arbitraje en diversas revistas especializadas. Asociado del Área de Arbitraje de Miranda \& Amado. Contacto: jrepetto@mafirma.com.pe.

Nota del Editor: El presente artículo fue recibido por el Consejo Editorial de THËMIS-Revistas de Derecho el día 10 de mayo de 2017 y, aceptado el 05 del agosto de 2017. 


\section{INTRODUCCIÓN}

La complejidad de los negocios ha generado la necesidad de estar atentos a nuevas y distintas fórmulas contractuales, las mismas que pueden tener impacto en la forma que se resuelven las controversias derivadas de dichos acuerdos. En la actualidad, un negocio puede estar compuesto no sólo de múltiples partes sino también de múltiples contratos los cuales pueden ser formalizados en diversos documentos escritos. Además, un negocio puede involucrar la suscripción de contratos sucesivos. Si bien el mejor escenario es que todos los contratos tengan cláusulas de solución de controversias similares o que se contemple una misma cláusula aplicable a las controversias de todos los contratos, ello no ocurre siempre. Un ejemplo ayuda ilustrar la situación.

Una empresa dedicada a la exploración y explotación de minerales ha realizado exploraciones en una determinada zona y ha encontrado grandes recursos minerales. Luego de evaluar la factibilidad económica del proyecto y haber obtenido las concesiones correspondientes por parte de la autoridad, como parte de sus operaciones decide construir una planta de procesamiento de minerales. Para ello, la corporación minera suscribe un contrato de diseño con una importante empresa de ingeniería y construcción para que elabore la ingeniería de detalle de la planta de procesamiento de minerales. Ese contrato contenía un "convenio arbitral CCI". Una vez entregada la ingeniería de detalle, la empresa minera decide suscribir un contrato de obra con la misma constructora para que se encargue de la construcción de la planta. La minera exige que la matriz de la constructora suscriba un contrato de garantía en virtud del cual asegure ante la minera el cumplimiento de todas las obligaciones de la constructora. Ninguno de esos dos contratos contiene una cláusula de solución de controversias. Luego de entregadas las obras, la constructora suscribe un contrato con la minera para que se encargue del mantenimiento de la planta de procesamiento de minerales. Resulta que, al año de terminada la construcción, la planta explota. Ante ello, la minera reclama ante la constructora que la planta no fue construida conforme a los estándares de la industria.

Si el contrato de construcción no contenía un convenio arbitral, pero el contrato de ingeniería sí y los reclamos provienen del contrato de construcción, ¿la empresa minera puede demandar en sede arbitral a la constructora? ¿a la matriz también? ¿qué ocurría si el contrato de construcción tiene una cláusula de solución de controversias ante el Poder Judicial? ¿Qué sucedería si el contrato de construcción tiene una cláusula de solución de controversias en una institución arbitral distinta a la del convenio arbitral o si es ad-hoc?

Justamente en el contexto de múltiples contratos o de un grupo de contratos, estas son preguntas que suelen formularse y que complican, o en todo caso, hacen más compleja, la solución de las controversias. ¿El convenio arbitral de un contrato puede comprometer las controversias de otros contratos?

Las circunstancias de cada caso son distintas y las controversias que se deriven de un contrato pueden impactar en el otro. Generalmente cada contrato tiene una cláusula de solución de controversias, las mismas que pueden ser diferentes e incluso incompatibles entre sí. Esto, obviamente, puede generar problemas en cuanto a la necesidad de activar más de un procedimiento de solución de controversias. Existirán procedimientos paralelos o procedimientos en los que la discusión sea la misma. En ese contexto, si existe un convenio arbitral en solo un contrato se pueden someter a arbitrajes las controversias de los demás contratos que no contienen un convenio arbitral. ¿Qué ocurre si el otro tiene una cláusula de foro judicial?

Esos problemas no se limitan al ejemplo antes descrito, sino que es parte de un problema real y cotidiano en la práctica del arbitraje internacional. Philippe Leboulanger, un reconocido comentarista, explica que:

"arbitral practice has witnessed the development of complex arbitrations as well as the specific procedural difficulties inherent thereto. A great source of such problems can be found in the large number of interrelated agreements involved in the performance of major projects, namely in the engineering, construction, raw materials, mining and oil sectors. These complex contractual relationships may give rise to parallel arbitrations and to situations in which the unity of the arbitral proceedings may be affected by the multiplicity of issues, agreements or parties involved in a certain dispute. However, international arbitration, which was tailored to the traditional two-party model involving a single contract, does not always provide satisfactory solutions to the procedural problems brought up by complex arbitrations"1.

\footnotetext{
LEBOULANGER, Philippe. "Multi-Contract Arbitration". En: Journal of International Arbitration 13. Kluwer Law International. 1996. p. 43. Sobre el mismo tema, Hanoatiau refiere que: "frequently happens that an economic transaction involves more than one contract. If one or more disputes arise between parties to one of the agreements of the contractual struc-
} ture, can all those who have participated in the economic transaction - or at least several of them, parties to different 
Lo anterior también es advertido por el profesor Gary Born, quien acertadamente indica que:

"Disputes sometimes arise where the parties have entered into a number of different agreements, either simultaneously or consecutively, each with (or sometimes without) a separate dispute resolution mechanism. This can create procedural difficulties, with the potential for parallel or overlapping arbitrations and litigations under different dispute resolution clauses. It also gives rise to questions of whether an arbitration clause in one contract applies to disputes under the provisions of another contract"2.

Sin duda, la situación es compleja e involucra soluciones creativas. Como bien sostienen los profesores Fouchard, Gaillard \& Goldman, una de las áreas más complicadas del arbitraje es determinar qué materias se encuentran comprometidas por un convenio arbitral en el contexto de grupos de contratos $^{3}$. Los autores precisan que cuando se suscriben diversos contratos que parecen estar conectados, pero solo algunos contienen cláusulas arbitrales, no es claro si las partes quisieron someter a arbitraje todo el esquema contractual a arbitraje ${ }^{4}$.

Así como existe la posibilidad de extender el convenio arbitral a una parte que no lo firmó (sobre lo cual se ha escrito mucho), otro problema recurrente y que a menudo pasa desapercibido es la posibilidad de extender un convenio arbitral de un contrato a otro contrato. Tribunales arbitrales y cortes judiciales han discutido si un convenio arbitral contenido en un contrato pueda ser extendido o su alcance puede incluir las controversias de otros contratos, con lo cual es relevante estar atentos a las corrientes jurisprudenciales más importantes. Por ello, en el presente artículo nos proponemos abordar si un convenio arbitral puede alcanzar a las controversias derivadas de otros contratos en el contexto de múltiples contratos.

La estructura del presente artículo es la siguiente: En la sección II se discuten algunas premisas básicas y generales con respecto al arbitraje en el contexto de múltiples contratos. En la sección III se describen los casos más resaltantes de arbitrajes con múltiples contratos. En la sección IV se describen los casos más resaltantes de arbitrajes con más de dos partes y múltiples contratos. Por último, en la sección $\mathrm{V}$ se presentan algunas conclusiones sobre este trabajo.

\section{PREMISAS BÁSICAS SOBRE EL ARBITRAJE EN EL CONTEXTO DE MÚLTIPLES CONTRATOS}

A diferencia de un arbitraje en el cual existe un único contrato, en un arbitraje con múltiples contratos la solución de las controversias es mucho más compleja y dependerá de las circunstancias propias de cada caso en concreto. Para aproximarnos a entender los problemas clásicos que se discuten en un arbitraje con múltiples contratos es necesario abordar una serie de cuestiones preliminares. En particular, en esta sección desarrollaremos: (A) la importancia del consentimiento en el arbitraje con múltiples contratos, (B) la conexión entre los contratos, (C) compatibilidad de las cláusulas arbitrales.

\section{A. Importancia del consentimiento en el arbi- traje con múltiples contratos}

El convenio arbitral es un contrato y fruto de la autonomía de voluntad de las partes ${ }^{5}$. El consentimiento es la pieza primordial que debe regir el análisis respecto a si un convenio arbitral puede ser extendido a otro contrato del esquema de múltiples contratos.

Mantilla Serrano coincide en ello y afirma que cualquier tribunal arbitral o corte que pretenda aplicar la doctrina del grupo de contratos debe determinar si las partes consintieron o no someter sus controversias a arbitraje ${ }^{6}$. Añade Mantilla Serrano que "without consent, no amount of connections between multiple parties and multiple contracts would be sufficient to give an arbitral tribunal jurisdiction"7.

contracts - be brought together in one single arbitration, before one single arbitral tribunal, which will then have to decide all their disputes? And may an arbitral tribunal hearing a dispute that arises principally from a specific contract decide issues arising from connected agreements entered into by the same parties, possibly alongside other contractors?". HANOTIAU, Bernard. "Complex Arbitrations: Multiparty, Multicontract, Multi-Issue and Class Actions". International Arbitration Law Library. Kluwer Law International. 2006. p. 101.

2 BORN, Gary. "International Commercial Arbitration". Segunda Edición. Kluwer Law International. 2014. p. 1370.

3 FOUCHARD, Philippe; GAILLARD, Emmanuel y Berthold GOLDMAN. "Fouchard, Gaillard \& Goldman on International Commercial Arbitration". Kluwer Law International.1999. 518.

4 FOUCHARD, Philippe; GAILLARD, Emmanuel y GOLDMAN, Berthold. Ibídem.

5 BORN, Gary. Óp. cit. p. 1317.

6 MANTILLA SERRANO, Fernando. "Multiple Parties and Multiple Contracts: Divergent or Comparable Issues?" En: Multiparty Arbitration, Dossiers of the ICC Institute of World Business Law 7. Kluwer Law International. 2010. p. 25.

7 MANTILLA SERRANO, Fernando. Ibídem. 
Al respecto, como bien sostiene Gary Born, el aspecto neurálgico para evaluar si un convenio arbitral puede ser extendido a otro contrato relacionado es la intención de las partes ${ }^{8}$. Sobre el particular, Bernard Hanotiau refiere acertadamente que:

"arbitration is consensual by nature. The arbitrators exclusively derive their jurisdiction from the will of the parties, expressed in an arbitration agreement. Any answer to the questions raised above must therefore start from an interpretation of the intent of the parties in the case in question, such as it is expressed in the arbitration clause" .

Lo anteriormente descrito tiene connotaciones prácticas relevantes. Mientras que un juez tiene, en principio, competencia para resolver en un mismo procedimiento las controversias de más de un contrato; un árbitro no. El árbitro emana su competencia del pacto de las partes ${ }^{10}$. En principio y salvo lo que se desarrollará en las secciones siguientes, sin consentimiento un árbitro no puede solucionar las controversias derivadas de otro contrato que no contiene un convenio arbitral compatible.

El escenario ideal y que solucionaría todos los problemas que describiremos a lo largo de este trabajo, es que las partes hayan pactado una cláusula arbitral "global". Un ejemplo de ello, es que el convenio arbitral conste en un documento separado e independiente que especifique y liste todos los contratos suscritos por las partes que se someterán a arbitraje. Otra opción es que las partes pacten una cláusula en alguno de los contratos celebrados, en el que se detalle que las controversias de todos los contratos suscritos entre las partes serán sometidas a arbitraje.
Sobre el particular, el profesor Gary Born explica que:

"International commercial agreements sometimes provide expressly for a single, unitary dispute resolution mechanism to govern all of the parties' various contractual relations. If such a global dispute resolution provision exists, either in a stand-alone agreement (providing an arbitration mechanism for disputes in a series of related substantive contracts) or as a clause in a single umbrella agreement, then application of the clause to disputes arising under several contracts is not controversial: there is no reason that an arbitration clause in one contract cannot encompass disputes or claims under another contract, provided that this is what the parties agreed. Frequently, however, the parties' drafting efforts lack this foresight, and disputes arise regarding the scope and interaction of overlapping or parallel dispute resolution provisions" ${ }^{\prime 1}$.

La fórmula global que ahorraría muchos problemas no siempre se pacta en la práctica y, por ello, es necesario analizar los convenios arbitrales. Para ello es sumamente relevante revisar el lenguaje empleado en las cláusulas arbitrales, ya que las partes son libres de establecer qué controversias pueden involucrar o abarcar un convenio arbitral.

En abstracto y en base al lenguaje utilizado, los contratantes son libres de definir qué tipos de controversias pueden ser sometidas a arbitraje y pactarlo así en su cláusula arbitral. Las partes podrán elegir someter exclusivamente las controversias de una etapa de la vida del contrato o pueden someter a arbitraje las controversias acerca de la validez, ejecución o terminación, incluyendo claro está la interpretación del acuerdo.

BORN, Gary. Óp. cit. p. 1374.

9 HANOTIAU, Bernard. "Complex Arbitrations: Multiparty, Multicontract, Multi-Issue and Class Actions". International Arbitration Law Library. Kluwer Law International. 2006. p.229.

10 Hanotiau explica que: "given the contractual nature of arbitration, the arbitrator does not have, with respect to multiparty arbitration, all the powers at the disposal of a judge. In the absence of an agreement between the parties (either express, or by reference to arbitration rules which authorise it, or implied), neither the tribunal, nor the institution that handles the arbitration, is entitled to consolidate ipso iure separate arbitration proceedings. It appears however that this lack of imperium does not prevent the arbitrator from deciding in certain cases, at the request of one of the parties to the arbitration, to stay the proceedings if the arbitrator judges them to be irregular, as long as another party to the contract and to the arbitration agreement has not been joined by the claimant, in the case, for example, that the parties' obligations are indivisible. The arbitrator could even invite the claimant, if the defendant so requests, to introduce parallel arbitration proceedings against a third party heavily implicated in the economic transaction by virtue of an interrelated contract. The arbitrator could then request the merger of the two proceedings if the applicable arbitration rules so permit - if, for example, the arbitrator finds that the failure to join that party is motivated purely by a strategy of obstruction.

Indeed, if the claimant does not comply, the arbitrator could not force him to do so, but he would be entitled to draw the resulting adverse inferences. The same situation can be found when the arbitrator invites one party to the arbitration, in response to a claim for provisional or conservatory measures, to refrain from carrying out an attachment, or not to release funds, or alternatively to submit a document being held by himself or by a third party. It is indeed another basic principle of international commercial arbitration that the parties have the duty to cooperate in good faith in the performance of their agreement as well as in the arbitral proceedings, e.g., at the stage of the constitution of the arbitral tribunal." HANOTIAU, Bernard. Ibid. pp. 233-234.

11 BORN, Gary. Óp. cit. p. 1370. 
Las partes también pueden excluir algún tipo de controversia específica. Por ejemplo, pueden acordar que no se sometan a arbitraje las discusiones de extensión de plazo en un contrato de obra o las controversias extracontractuales (de enriquecimiento sin causa, por ejemplo) que se encuentren relacionadas con la ejecución del contrato.

Lo importante a efectos de este trabajo, es que las partes pueden utilizar fórmulas de redacción amplias que puedan incluir la aplicación del convenio arbitral a contratos relacionados. Las partes pueden acordar una determinada receta de redacción amplia de su convenio arbitral para que este se pueda extender a otros contratos ${ }^{12}$. Así, por el uso de la palabra "relacionada", "que guarden relación", "en conexión con" en una usual fórmula de redacción del convenio arbitral podría dar a entender que las partes quisieron someter a arbitraje no solo las controversias del contrato, sino además las de cualquier otro contrato relacionado o que guarde relación con dicho contrato.

En consecuencia, si de lo que se trata es determinar si un convenio arbitral puede ser extendido a otros contratos "relacionados", entonces se deberá estar sumamente atento a la existencia de un "convenio arbitral global" (escenario ideal) o un convenio arbitral con una redacción amplia.

Por último y no por ello menos importante, un aspecto que no debe ser descuidado al momento de interpretar el alcance del convenio arbitral, es el derecho aplicable que regulará dicho análisis.

La definición del derecho aplicable a la interpretación no es un tema menor y puede tener implicancias prácticas en cómo se debe interpretar el convenio arbitral. Mientras que algunos ordenamientos jurídicos son fieles a la letra del contrato, existen otros que son más permeables a elementos extratextuales estando autorizadas las partes a probar en contra del sentido literal de las palabras.

La interpretación del convenio arbitral se rige, en primer lugar, por el derecho elegido por las partes, es decir, la norma que las partes hayan acordado específicamente para regular el convenio arbitral ${ }^{13}$. Vale mencionar que, como consecuencia del principio de separabilidad, se entiende que el derecho aplicable al contrato no es necesariamente el mismo que el del convenio arbitral $^{14}$. Es decir, si se conviene en el contrato que este se regule conforme al derecho español, esto no implica necesariamente que la validez e interpretación del convenio arbitral se rija bajo el derecho español.

Es aceptado que el derecho aplicable a la interpretación del convenio arbitral debe ser el mismo que el derecho que regula la validez del convenio

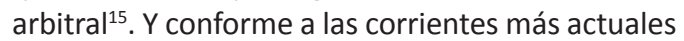
y aceptadas, el derecho que regula la validez del convenio arbitral es el derecho de la sede del arbitraje $^{16}$. En consecuencia, el derecho bajo el cual se debe interpretar el convenio arbitral, es el derecho de la sede del arbitraje (salvo pacto en contrario). Por ejemplo, si se acuerda que el contrato se rige bajo el derecho español, pero la sede del arbitraje es México D.F. y no se establece que el convenio arbitral se regula por un determinado derecho, entonces la validez e interpretación de dicho convenio arbitral debe ser analizada al amparo del derecho mexicano.

\section{B. Conexión entre los contratos}

\section{Importancia de las razones económicas}

Otro aspecto preponderante al momento de analizar si un convenio arbitral puede ser extendido a otro contrato, es la vinculación entre ambos contratos. Generalmente, se entiende que es posible la extensión del convenio arbitral a otros contratos

BORN, Gary. Ibid. p. 1374.

3 BORN, Gary. Ibid. pp. 491 y ss.

14 BORN, Gary. Ibid. pp. 475-476: "One of the most direct consequences of the separability presumption is the possibility that the parties' arbitration agreement may be governed by a different law than the one governing their underlying contract. That is, although the parties' underlying contract may be governed expressly, or impliedly, by the laws of State A, the associated arbitration clause is not necessarily governed by State A's laws, and may instead be governed by the laws of State B or by principles of international law. This result follows, almost inevitably, from the separability presumption, which postulates two separable agreements, which can in principle be governed by two different legal regimes. As one arbitral award observed, "an arbitration clause in an international contract may perfectly well be governed by a law different from that applicable to the underlying contract." The separability doctrine does not mean that the law applicable to the arbitration clause is necessarily different from that applicable to the underlying contract. It instead means that differing laws may apply to the main contract and the arbitration agreement. Despite this possibility, in many cases, the same law governs both the arbitration agreement and the underlying contract."

15 BORN, Gary. Ibid. p. 635: "Choice-of-law analysis is also necessary to select the law applicable to interpretation of an international arbitration agreement. Many (but not all) jurisdictions apply the same law to the interpretation of an arbitration agreement as to its formation and substantive validity."

16 BORN, Gary. Ibid. pp. 509 y ss. 
en base a consideraciones económicas comunes entre los contratos.

Como bien informa Hanotiau citando a F.X. Train, los contratos están relacionados cuando están vinculados uno al otro en una misma relación económica o dependencia funcional ${ }^{17}$.

Mantilla Serrano sostiene que debe existir un lazo económico que crea un vínculo entre los múltiples contratos $^{18}$. Agrega el reconocido abogado que: "without this economic link to bind the contracts together, there would be little reason to consider that they should share a common arbitration agreement"19.

En suma, los aspectos económicos son parte esencial para determinar si dos o más contratos se encuentran relacionados.

2. Factores que se toman en cuenta para evaluar la conexión entre los contratos

Ahora bien, cabe preguntarse qué elementos económicos se deben tomar en cuenta para evaluar si dos contratos están relacionados. ¿Basta qué se celebren dos contratos entre las mismas partes para que se pueda extender el convenio arbitral de uno al otro?

Una respuesta a lo anterior, se encuentra en lo postulado en términos abstractos por F.X. Train. El referido autor sostiene que existen dos tipos de contratos que se encuentran relacionados ${ }^{20}$. En primer lugar, aquellos grupos de contratos que coexisten para lograr un objetivo común, como puede ser el caso de: (i) un acuerdo marco y contratos de implementación de dicho acuerdo marco; (ii) un contrato principal y un contrato accesorio para financiar la transacción principal; (iii) un grupo de contratos de igual nivel y que conjuntamente logran un objetivo común. En segundo lugar, se encuentran (i) aquellos grupos de contratos que se encuentran unidos en relación de sustitución o (ii) dos contratos sucesivos entre las mismas partes, en el cual el posterior impacta en el primero modificándolo o extinguiéndolo (sea a través de una transacción, novación o mutuo disenso).
F.X. Train precisa que, por ejemplo, los contratos sucesivos entre las mismas partes que tienen la misma naturaleza no se encuentran relacionados. Se trata de contratos que se celebran, generalmente, uno luego de haberse terminado otro. Por ende, son contratos que, a diferencia de los contratos conectados, se suelen celebrar en distintos momentos temporales. Este sería el caso, por ejemplo, de la celebración de sucesivos contratos de compraventa de bienes. Si bien para el referido autor dichos contratos no se encuentran relacionados, autores de la talla de Gary Born y Fouchard, Gaillard \& Goldman presentan evidencia que en esos contextos también se ha logrado extender el convenio arbitral de un contrato a otro contrato sucesivo ${ }^{21}$.

Por su parte, la jurisprudencia francesa ha considerado, entre otros, que los siguientes grupo de contratos están interrelacionados o coligados: (i) cuando se celebran contratos sucesivos con prestaciones recíprocas (como es el caso de varias compraventa de bienes), (ii) cuando existen convenios marcos y contratos de implementación (como es el caso de contratos de larga duración o contratos de distribución) y (iii) contratos que si bien están al mismo nivel y no existe un acuerdo marco, existe una relación económica conjunta que los une 22 .

En esa misma línea, Hanotiau sostiene que:

"the courts have uniformly concluded that if two agreements between the same parties are closely connected and one finds its origin in the other, or is the complement or the implementation of the other, the absence of an arbitration clause in one of the contracts does not prevent disputes arising from the two agreements from being submitted to an arbitral tribunal and decided together"23.

Según explica Hanotiau, el principal problema consiste en determinar si los contratos constituyen un todo indivisible, lo cual dependerá de la intención de las partes. Al respecto, Laurent Aynes explica que un todo indivisible significa que: "if each of the partial agreements exists only by the preceding and calls on the following; a will, unique in its aim, is expressed in a variety of complementary instru-

\footnotetext{
HANOTIAU, Bernard. Óp. cit. 219.

18 MANTILLA SERRANO, Fernando. Óp. cit. p. 19.

19 MANTILLA SERRANO, Fernando. Ibid. p. 19.

20 HANOTIAU, Bernard. Óp. cit. p. 219.

21 BORN, Gary. Óp. cit. pp. 1376-1379. FOUCHARD, Philippe; GAILLARD, Emmanuel y Berthold GOLDMAN. Óp. cit. pp. 305-306.

22 LEBOULANGER, Philippe. Óp. cit. pp. 49-50.

23 HANOTIAU, Bernard. Óp. cit. p. 281.
} 
ments. It is also the existence of a subordination that makes it possible to identify the group formed by a main contract and a sub-contract" 24 .

Si bien los elementos descritos en los párrafos anteriores son algunos elementos característicos para considerar que dos o más contratos se encuentran relacionados, en la descripción de la jurisprudencia (sección III) se desarrollarán algunos otros factores o casos en los cuales se ha considerado posible extender el convenio arbitral de un contrato a otro relacionado. En definitiva, las consideraciones económicas son una cuestión fáctica y deberá evaluarse caso por caso para determinar si existe conexión económica entre los contratos celebrados por las partes.

3. Las consideraciones económicas por sí solas no generan el consentimiento

Es importante mencionar que las consideraciones económicas para determinar la extensión de un contrato a otros son tan solo un elemento a evaluar, ya que también es importante tener en cuenta el consentimiento ${ }^{25}$. Mantilla Serrano explica que las cortes francesas han rechazado extender las cláusulas arbitrales de un contrato a otro por la sola y exclusiva razón de que los contratos formaban parte de una misma operación económica ${ }^{26}$. Se requiere pues, además, explicar el consentimiento a arbitrar antes descrito.

En algunos casos, sin embargo, se ha argumentado con éxito que el vínculo económico entre los contratos puede ser interpretado como una muestra de la común intención de las partes a efectos de la extensión del convenio arbitral de un contrato a otro relacionado. Lo anterior ha sido explicado, por ejemplo, en el caso Glencore resuelto por la Corte de Apelaciones de París ${ }^{27}$.

Los entretelones de esa decisión se remontan a que Glencore celebró un contrato de compraventa internacional de un cargamento de arroz con la empresa congolesa Afric. Dicho contrato tenía una cláusula arbitral que remitía las controversias a un arbitraje con sede París. Ante la existencia de una guerra en curso en el Congo, Afric y Glencore celebraron un segundo contrato en el cual Glen- core no entregaría el arroz en la fecha pactada y reembolsaría ciertos pagos a Afric. Este segundo contrato no tenía ninguna cláusula de solución de controversias.

Resulta que, surgida una controversia sobre la ejecución del segundo contrato, Afric inició un arbitraje y el tribunal arbitral consideró que tenía competencia sobre el segundo contrato. Glencore planteó un recurso de anulación ante la Corte de Apelaciones de París sobre la base que los árbitros no tenían competencia para pronunciarse sobre el segundo contrato.

La mencionada corte dictaminó de que, sobre la base de los hechos, ambos contratos tenían un vínculo económico estrecho lo cual revelaba que la implícita común intención de las partes era que necesariamente las disputas de ambos contratos se resuelvan mediante arbitraje.

En consecuencia, si bien no basta únicamente argumentar que un convenio arbitral de un contrato puede extenderse a otro sobre la base de la mera existencia de una vinculación económica entre los contratos, el elemento económico puede ser utilizado como un indicador relevante que la común intención de las partes era extender la cláusula de un convenio arbitral de uno de los contratos a otro.

Por ello, sin temor a exagerar, Mantilla Serrano afirma que: "the economic link between the contracts necessarily becomes a key consideration in determining the existence of a group of contracts" 28 .

\section{Compatibilidad}

Al existir múltiples contratos un problema adicional que se plantea es que cada uno de los contratos tenga su propia cláusula de solución de controversias. En un escenario de tan solo dos contratos, las opciones que pueden plantearse son múltiples y pueden ser resumidas de la siguiente manera:

- Ambos contratos tienen la misma cláusula arbitral.

- Los contratos tienen diferentes cláusulas arbitrales.

24 HANOTIAU, Bernard. Ibídem. Citando a Laurent Aynes.

25 El professor Gary Born sostiene que: "Of course, the ultimate touchstone is the intentions of the parties and the language of the arbitration clause, which may address the matter either expressly or by implication (by being drafted in either particularly broad or deliberately narrow terms)." BORN, Gary. Ibid. p. 1374. Por su parte, Mantilla Serrano precisa que: "finding the existence of an economic link between the parties is only one part of the puzzle; there must also be intent and consent." MANTILLA SERRANO, Fernando. Óp. cit. p. 19.

26 MANTILLA SERRANO, Fernando. Óp. cit. 2010. p. 19.

27 Société Glencore Grain Rotterdam BV v. Société Afric, Corte de Apelaciones de París, 23 de noviembre de 1999.

28 MANTILLA SERRANO, Fernando. Óp. cit. pp. 21-22. 
- Un contrato tiene una cláusula arbitral y el otro contrato tiene una cláusula de foro judicial.

- Un contrato tiene una cláusula arbitral y el otro no tiene una cláusula de solución de controversias.

La compatibilidad de las cláusulas de solución de controversias es un aspecto fundamental a considerar. Es decir, existe la posibilidad que en un mismo caso se presenten circunstancias en las cuales exista la posibilidad de un indicio fuerte de consentimiento y una relación económica suficiente, pero que las cláusulas de solución de controversias sean incompatibles, lo cual pueda frustrar la posibilidad de extender un convenio arbitral de un contrato a otro o que todas las controversias se tramiten en un mismo foro.

Con respecto a qué puede considerarse como compatible, el profesor Train indica que el test de compatibilidad pasa por preguntar cuál fue la voluntad de las partes ${ }^{29}$. En específico, se debe determinar si las partes querían renunciar a la extensión de una cláusula arbitral al introducir otra cláusula diferente en otro de los contratos del grupo. El profesor Train, citado por Hanotiau, refiere en el contexto de dos convenios arbitrales:

"Clauses will be considered incompatible if the difference relates to a fundamental element of the arbitration agreement: the institutional or ad hoc nature of the arbitration, the seat, the number of arbitrators, the appointment procedure. If, on the other hand, the difference relates to a secondary element (law applicable to the merits, steps to be taken before the initiation of the procedure, etc.), the clauses will be considered compatible" ${ }^{30}$.

En línea con lo indicado por el profesor Train, el análisis de compatibilidad de dos convenios arbitrales es una circunstancia delicada que trata de desentrañar la común intención de las partes, lo cual deberá analizarse caso por caso.

En principio, si existen controversias de dos contratos o las controversias involucran más de un contrato (cada contrato con su propio convenio arbitral), estas se pueden plantear en un mismo arbitraje, siempre y cuando los convenios arbitrales sean idénticos o compatibles y de los hechos del caso se puede entender que las partes tenían la intención de someter toda la operación a un tribunal arbitral ${ }^{31}$.

Como bien señala Fouchard, Gaillard y Goldman la mejor solución para evitar que dos tribunales arbitrales arriben a decisiones contradictorias derivadas de dos contratos es iniciar un solo arbitraje (en caso los convenios arbitrales sean compatibles) o consolidar dos o más arbitrajes existentes.

De hecho, algunos reglamentos arbitrales permiten a las partes consolidar arbitrajes entre las mismas partes que provengan de la misma relación jurídica y designen a la misma institución arbitral. Así, por ejemplo, el Reglamento de Arbitraje de la Cámara de Comercio Internacional dispone que:

\section{Artículo 10.-}

"La Corte podrá, a solicitud de una parte, consolidar dos o más arbitrajes pendientes bajo el Reglamento en un solo arbitraje, cuando: [...] c) si las demandas en los arbitrajes son formuladas bajo más de un acuerdo de arbitraje, los arbitrajes sean entre las mismas partes, las controversias en los arbitrajes surjan en relación con la misma relación jurídica, y la Corte considere que los acuerdos de arbitraje son compatibles" 32 .

Por su parte, existen algunas legislaciones, como la holandesa, que autorizan a las cortes consolidar procedimientos arbitrales que provengan de acciones relacionadas ${ }^{33}$

29 HANOTIAU, Bernard. Óp. cit. p. 269.

30 HANOTIAU, Bernard. Ibid. p. 269.

31 FOUCHARD, Philippe; GAILLARD, Emmanuel y GOLDMAN, Berthold. Óp. cit. p. 302: "it is generally legitimate to presume that by including identical arbitration clauses in the various related contracts, the parties intended to submit the entire operation to a single arbitral tribunal."

32 Reglamento de Arbitraje de la Cámara de Comercio Internacional, 2012.

33 The Arbitration Act, Code of Civil Procedure, Book IV, 1 December 1986, as amended 1 January 2015, Artículo 1046: "(1) In respect of arbitral proceedings pending in the Netherlands, a party may request that a third person designated to that end by the parties order consolidation with other arbitral proceedings pending within or outside the Netherlands, unless the parties have agreed otherwise. In the absence of a third person designated to that end by the parties, the provisional relief judge of the district court of Amsterdam may be requested to order consolidation of arbitral proceedings pending in the Netherlands with other arbitral proceedings pending in the Netherlands, unless the parties have agreed otherwise.". Sobre dicho artículo se ha comentado que: "By virtue of Article 1046 DCCP, arbitrations with related subjects can be consolidated in the Netherlands, unless the parties agree otherwise. Since consolidation as a rule considerably complicates arbitrations, excluding this possibility of consolidation in the arbitration agreement is usually recommended. This results primarily from the process that is to lead to consolidation of arbitrations. Once consolidation has been decided on in 
Como bien indica Born, cuando los convenios arbitrales son compatibles o con redacción idéntica, las Cortes de los Estados Unidos, Francia, Inglaterra, Suiza y Alemania, así como diversos tribunales arbitrales han considerado que un convenio arbitral de un contrato puede extenderse a otro contrato relacionado ${ }^{34}$.

\section{JURISPRUDENCIA SOBRE MULTI-CONTRATOS}

En esta sección se desarrollarán los principales casos en los cuales tribunales arbitrales se han enfrentado a un esquema de contratos múltiples.

A grandes rasgos, existen tres tipos de situaciones a las cuales se han enfrentado los tribunales arbitrales y las cortes judiciales: (A) cuando existe un convenio arbitral en un contrato y otro contrato del grupo no tiene una cláusula de solución de controversias; (B) cuando existe un convenio arbitral en un contrato y otro contrato del grupo tiene una cláusula judicial; (C) cuando dentro de los múltiples contratos existen convenios arbitrales cuya redacción no es similar.

\section{A. Cuando existe un convenio arbitral en un contrato y otro contrato del grupo no tiene una cláusula de solución de controversias}

La recopilación de estos casos está referida a que un contrato del grupo de contratos tiene un convenio arbitral y otro (u otros) contratos no tiene una cláusula de solución de controversias. Es decir, el otro contrato no tiene ni un convenio arbitral ni una cláusula de jurisdicción judicial.

\section{Decisión de la Corte Suprema de Francia del 30 de marzo de $2004^{35}$}

Este es, sin duda, uno de los casos más emblemáticos de extensión del convenio arbitral a otros contratos del grupo de contratos que no tienen cláu- sula de solución de controversias. Resulta que el 17 de octubre de 1990, UNI-KOD Sarl ("Uni-Kod") celebró un contrato de joint-venture con Ouralkali y otras tres empresas rusas para establecer UNI, una empresa dedicada a producir cosméticos por cinco años. Como parte de sus obligaciones, Uralkali se comprometió a aportar US\$ 5 millones los cuales serían destinados a comprar materiales y compuestos químicos. El contrato de joint-venture remitía las controversias derivadas de este contrato a la Comisión de Arbitraje de Comercio Exterior de la Cámara de Comercio e Industria de la Unión Soviética.

Menos de un mes de celebrado el contrato de joint-venture, Uni-Kod y Ouralkali suscribieron el denominado Contrato 1, bajo el cual Ouralkali adelantó a Uni-Kod el precio de compra de ciertos insumos y materiales químicos tal como lo establecía el contrato de joint-venture. Este contrato no contenía un convenio arbitral.

Resulta que el 20 de diciembre de 1996, Ouralkali inició un arbitraje contra Uni-Kod al amparo de la cláusula arbitral del contrato de joint-venture. En dicho arbitraje Ouralkaili reclamó la restitución de un millón y medio de dólares pagados bajo el Contrato 1. Por su parte, Uni-Kod también planteó reclamos contra Uni-Kod. En octubre de 1998, el tribunal arbitral emitió un laudo favorable a Ouralkali.

Ouralkali solicitó el reconocimiento del laudo ante las cortes francesas. El 5 de octubre de 1999, el Presidente de la Corte de Primera Instancia de París reconoció el laudo. Uni-Kod apeló la sentencia de reconocimiento ante la Corte de Apelaciones de París, alegando que el laudo que se pretendía reconocer derivaba del Contrato 1 , el cual no contenía un convenio arbitral y que el convenio arbitral del joint-venture no se podía extender al Contrato 1. La Corte de Apelaciones de París, en sentencia del 31 de mayo de 2001, determinó que el Contrato

principle, arbitrators still have to be appointed and agreements have to be made with regard to the rules which will apply to the consolidated proceedings. All of this is especially complex if the consolidated arbitrations were subject to different rules or regulations. Once consolidation has been ordered, proceedings involving three or more parties frequently ensue. Such proceedings may naturally become highly complex as a result thereof. In addition, the fact that a request for consolidation must be submitted to the state courts may compromise confidentiality of the (existence of) arbitral proceedings. Against this background, the possibility of consolidation should only be considered in the event of legal relationships that are very closely related, because in such cases it might be strongly preferable to have different disputes resolved at the same time and by the same arbitrators to avoid conflicting decisions. Unless the parties have excluded the possibility of consolidation, consolidation must be realized by means of proceedings before the President (voorzieningenrechter) of the Amsterdam District Court. The President may also order partial consolidation." MARNIX LEIJTEN, Marc Ynzonides y Bommel van der Bend. "A Guide to the NAI Arbitration Rules: Including a Commentary on Dutch Arbitration Law". Kluwer Law International. 2009. pp. 24-25.

34 BORN, Gary. Óp. cit. pp. 1371-1372.

35 France E5. UNI-KOD sarl v. Ouralkali, Corte de Apelación [Court of Appeal]. 31 de mayo de 2001. En: Albert Jan van den Berg (editor). Yearbook Commercial Arbitration 2001 - Volumen 26. Kluwer Law International. 2001. pp. 1136-1140. 8-9. France E6, S.a.r.I. UNI-KOD (Francia) v. Ouralkali (Russian Federation), Corte de Casación [Supreme Court]. 539. F-P. 30 de marzo de 2004. En: Albert Jan van den Berg (editor). Yearbook Commercial Arbitration 2005. Volumen 30. Kluwer Law International. 2005. pp. 1200-1203, 3. 
1 y el contrato de joint-venture eran inseparables y que garantizaban la viabilidad económica de la operación. Adicionalmente, la Corte identificó que el convenio arbitral del contrato de joint-venture era lo suficientemente amplio como para considerar aplicable a las controversias del Contrato 1. En palabras de la mencionada Corte:

"Contract 1 of 2 November 1990 is complementary to the joint venture agreement of 17 October 1990. These two agreements are unseverable so that to guarantee the economic viability of the operation, notably as they supply UNI-KOD with currency against its investment in Russia. We must therefore deem that the parties considered the arbitration clause in the contract of 17 October 1990 as tacitly but necessarily included in Contract 1 .

Also, the arbitration clause is formulated in very general terms that provide, as mentioned above, that all disputes and differences arising under the joint venture agreement or in relation thereto, such as Contract 1 , are referred to arbitration" ${ }^{36}$.

A pesar de que el laudo fue reconocido por la Corte de Apelaciones de París, Uni-Kod apeló ante la Corte de Casación de Francia. Sin embargo, no tuvo éxito. La Corte de Casación de Francia coincidió en la posición de la Corte de Apelaciones de París y determinó que:

"Nor did the [Court of Appeal] misrepresent Art. 18 of the agreement of 17 October 1990 when it held by a sovereign evaluation of the arbitration clause - whose very general terms required interpretation - that the two agreements were unseverable in order to guarantee the economic viability of the operation, and that the referral to arbitration concerned all disputes and differences arising under the joint venture agreement or in relation thereto, such as Contract 1, so that the arbitration clause was tacitly but necessarily included in Contract $1^{\prime \prime 37}$

Como se puede apreciar, la lección que deja este caso es que si los contratos se encuentran intrínsecamente relacionados (al punto que ambos forman y garantizan la viabilidad económica de la transacción) y la cláusula arbitral es amplia, entonces se puede extender el convenio arbitral de un contrato al contrato que no tiene convenio arbitral.

\section{Caso $\mathrm{CCl} 8420^{38}$}

En este caso, un agente sirio celebró un contrato de agencia con un distribuidor italiano, en virtud del cual el agente se comprometió a promocionar los productos del distribuidor italiano. Este contrato tenía un convenio arbitral que remitía las controversias a un arbitraje ICC. Cuatro años después de la celebración del mencionado acuerdo, las partes celebraron dos contratos secundarios: un Protocol Agreement y un Customs clearance letter, los mismos que no tenían un convenio arbitral.

Suscitada una serie de controversias entre las partes, el agente sirio inició un arbitraje contra el distribuidor italiano, alegando incumplimientos contractuales no sólo del contrato de agencia sino también de los otros dos contratos. El distribuidor italiano se opuso manifestando que no existía una cláusula arbitral en dichos contratos y que el convenio arbitral del contrato de agencia no se podía extender.

El Tribunal Arbitral que resolvió esta controversia precisó que en aquellos casos en los cuales se había extendido un convenio arbitral a contratos secundarios, era porque se trataba de contratos secundarios modificatorios o que completaban el principal o se trataban de contratos que estaban significativamente relacionados con el principal, en el sentido que su futura existencia o necesidad se encontraban anticipadamente contemplados en el contrato principal ${ }^{39}$. En ese sentido, el Tribunal Arbitral explicó que cuando los contratos secundarios son solo una implementación, modificación o cumplimiento de la relación contractual principal o constituyen una consecuencia necesaria del contrato, entonces se entiende que se puede extender el convenio arbitral ${ }^{40}$.

Luego de ello, el Tribunal Arbitral pasó a analizar las circunstancias propias del caso en concreto y determinó que el Protocol Agreement ni la Customs clearance letter no eran una implementación del contrato de agencia. En particular, el Tribunal Arbitral explicó que:

"The 'principal' agreement, i.e. the agency, is quite a different relationship than the one created by the Protocol and the Customs clearance letter. The two 'secondary' contracts [...] rep-

36 France E5, UNI-KOD sarl v. Ouralkali. Ibídem.

37 France E6, S.a.r.I. UNI-KOD (Francia) v. Ouralkali (Federación Rusa). Óp. cit. Loc. cit.

38 Agent v. Supplier. Partial Award. ICC Case 8420. 1996. En: Albert Jan van den Berg (editor), Yearbook Commercial Arbitration 2000. Volumen 25. Kluwer Law International. 2000. pp. 328- 340.

39 Agent v. Supplier. Ibid. p. 46.

40 Agent v. Supplier. Ibid. p. 48-49. 
resent neither a fulfillment nor an amendment of the previous contractual relationship i.e. the agency agreement, but something completely new, which gives the parties different duties and obligations which are not directly connected with the agency. One thing is to promote the sale of products, another thing is 'renting' premises and personnel for carrying out a contract of sale and contraction as well as taking care of shipments and customs clearance. Neither is there any evidence that such two 'secondary' contracts were very closely connected with the 'principal', in the sense that at the time of the signing of the agency agreement all parties were aware of the fact that such contracts would necessarily imply the future signing of the above mentioned two secondary contracts.[...] The Protocole and the Customs clearance letter do not form an economic unit ('unité economique') with the agency agreement, neither are they an amending contract ('contrat accessoire') to the latter, nor are they a necessary and previously agreed consequence of the agency agreement" ${ }^{\prime 1}$.

Este caso es una muestra de que cuando los contratos no tienen conexión significativa, entonces no se puede extender el convenio arbitral al otro.

\section{Caso $\mathrm{CCl} 11440^{42}$}

En el caso bajo comentario, en el año 1998 dos partes suscribieron un Master Agreement en virtud del cual una parte alemana se comprometió a vender un negocio a una parte italiana. Dicho contrato contenía un convenio arbitral ICC. Según el Master Agreement el precio sería determinado sobre la base del Consolidated Financial Statement (CFS). Existieron controversias respecto al mencionado documento, lo que motivó que las partes suscribieran un Settlement Agreement, el cual no contenía un convenio arbitral.

Luego de ello se suscitaron una serie de controversias derivadas del Master Agreement, ya que el comprador italiano alegaba que el vendedor había violado ciertas representations, warranties y otras obligaciones. Por ello, el comprador inició un arbitraje ante la $\mathrm{CCl}$. En el arbitraje, el vendedor planteó también una serie de reclamos. Según el comprador los reclamos del vendedor se basaban en el Settlement Agreement, el cual no contenía un convenio arbitral. El Tribunal Arbitral que cono- ció la controversia no dudó en extender el convenio arbitral del Master Agreement al Settelement Agreement.

\section{Caso $\mathrm{CCl} 7929^{43}$}

En este caso una empresa estadounidense dedicada a la producción de bienes de construcción celebró un Partnership Agreement con una empresa finlandesa y su subisidiaria para producir y vender una serie de productos. Dicho contrato contenía un convenio arbitral que remitía sus futuras controversias a un arbitraje administrado bajo los auspicios de la Cámara de Comercio Internacional con sede en Zurich, Suiza. Adicionalmente, las tres partes celebraron un Cooperation Agreement, el cual no contenía una cláusula arbitral ni una cláusula de jurisdicción judicial.

Resulta que en determinado momento de la ejecución de los contratos se suscitó una controversia y la empresa finlandesa y su subsidiaria iniciaron un arbitraje ante la $\mathrm{CCl}$ sobre la base del convenio arbitral contenido en el Partnership Agreement. Ante ello, la empresa estadounidense objetó la competencia de los árbitros para pronunciarse respecto al Cooperation Agreement, porque el mismo no tenía un convenio arbitral. Los demandantes argumentaron que sí se podían someter a arbitraje las controversias del Cooperation Agreement, ya que si bien el mismo no tenía un convenio arbitral, el Partnership Agreement sí y ambos contratos conformaban parte un esquema contractual único.

A través de un laudo parcial, el tribunal arbitral resolvió que: (a) un convenio arbitral de un contrato puede extenderse a otro si ambos conforman un esquema contractual único, y (b) decidió postergar la decisión de si ambos contratos conforman un esquema contractual único para una fase posterior del arbitraje. Lo relevante de esta decisión es, pues, que los árbitros decidieron amparar la posibilidad de extender el convenio arbitral de un contrato a otro si ambos configuran un esquema contractual único. En palabras de los árbitros:

'The phrase 'unified contractual scheme' in the present context appears to us to reflect the position frequently encountered in international arbitration with, in the words of Craig, Park \& Paulsson, 'complex situations where numerous contractual documents relate to one organic relationship'. Pri-

\footnotetext{
$41 \quad$ Agent v. Supplier. Ibid. 51-52.

42 Buyer (Italia) v. Seller (Alemania). Final Award, ICC Case 11440-2003. En: Albert Jan van den Berg (editor). Yearbook Commercial Arbitration 2006. Volumen 31. Kluwer Law International. 2006. pp. 127 - 147

43 Manufacturer, Subsidiary v. Building supplier. Interim Award. ICC Case 7929. 1995. En: Albert Jan van den Berg (editor) Yearbook Commercial Arbitration 2000. Volume 25. Kluwer Law International. 2000. pp. 313-323.
} 
ma facie, it also appears to describe the situation in this case, to the extent that there is a main contract (the Partnership Agreement) which creates a business relationship, and other contracts (notably the Cooperation Agreement) making changes of one sort or another in that relationship. It is generally considered desirable that disputes relating to obligations arising from the relationship as a whole, i.e. under several contractual documents, should be submitted to one, single judicial authority: 'it appears inappropriate for different jurisdictions to deal with necessarily interrelated issues in a piecemeal and potentially inconsistent manner [...].

In the present case, then, the real question is whether the two agreements, that of 1986 and that of 1987, either alone or in conjunction with other contractual arrangements between the parties, constitute what is described as a 'unified contractual scheme'. From what we know of the case so far, it seems that the Cooperation Agreement did not stand alone. It appears to have been one of a series of agreements concluded between the parties, as part of a business relationship which began in 1985 and which inter alia expressed the wish to settle any disputes by arbitration. Whilst the meaning and effect of the Cooperation Agreement remain to be determined at a later stage in these proceedings, the Agreement itself apparently envisaged that some relationship between the parties would continue, since it stated that the parties would 'continue their existing relationship of mutual respect and business development.' (footnote omitted) This would seem to point to a 'unified contractual scheme' or, to put it in another way, to disputes which have their 'origin or genesis' in the Partnership Agreement. However[...], it is too soon for us to form any opinion as to whether or not their is a unified contractual scheme" ${ }^{\prime 4}$.

Como vemos, en este caso se decidió que en abstracto si se probaba la existencia de un esquema contractual único, el Tribunal Arbitral podía conocer las controversias derivadas del contrato que no contenía un convenio arbitral.

5. Caso Glencore (Decisión de la Corte de Apelaciones de París 23 de noviembre de 1999)

En el caso Glencore, explicado en la sección II anterior, la Corte de Apelaciones de París extendió el convenio arbitral contenido en el contrato de compraventa al segundo contrato, porque en su opinión ambos contratos constituían una relación contractual. En particular, la Corte apreció que dichos contratos se celebraron a las pocas semanas y estaban intrínsecamente conectados, ya que el primer contrato versaba sobre sobre la entrega de una determinada mercadería y el segundo contrato versaba sobre la devolución de la misma mercadería ${ }^{45}$.

Además, para la Corte el segundo contrato tenía un vínculo económico estrecho con el primero, ya que era una continuación del primero y una consecuencia del primero ${ }^{46}$.

6. Fletamentos Maritimos SA v. Effjohn International $\mathrm{BV}^{47}$

En este caso Fletamentos Maritimos S.A. suscribió un contrato de cooperación con Effjohn International BV para establecer un negocio de cruceros. Este contrato contenía un convenio arbitral con sede en Inglaterra. Posteriormente, las partes compraron un barco en nombre de una empresa de Bermuda, $X$. Resulta que surgió una controversia porque Fletamentos Maritimos consideraba que Effjohn International BV no había pagado la parte que le correspondía con respecto a la adquisición del barco. Por ello, Fletamentos Marítimos inició un arbitraje y Effjohn International BV sostuvo que el tribunal arbitral no tenía competencia para pronunciarse sobre dicha adquisición, ya que provenía de un contrato que no tenía un convenio arbitral.

La Corte sostuvo que Fletamentos Maritimos S.A. no tenía razones para oponerse al arbitraje, ya que la compra del barco fue celebrada en el contexto del contrato de cooperación el cual contenía un convenio arbitral.

7. Decisión de la Corte de Apelaciones de París del 21 de junio de $1990^{48}$

En este caso la Corte de Apelaciones de París consideró que no era posible extender el convenio arbitral de un contrato a otro porque eran dos contratos que no tenían conexión. Los hechos de este caso se remontan a un contrato de distribución suscrito entre Honeywell Bull (una empresa

44 Manufacturer, Subsidiary v. Building supplier. Ibid. 31 y 37.

45 Société Glencore Grain Rotterdam BV v. Société Afric. Óp. cit.

46 Ibídem.

47 Fletamentos Maritimos SA v. Effjohn International BV (1996. 2 Lloyd's Rep 304 QB). En: "Jurisprudence - England", ASA Bulletin: Association Suisse de l'Arbitrage. Kluwer Law International 1997. Volume 15. pp. 130-135.

48 First Supplement Ch. 1991 Rev. Arb. 96. 
francesa) con Sistemas Ancater (una empresa venezolana). Este contrato tenía como objetivo que la empresa francesa le otorgue la distribución exclusiva de productos de computación en Venezuela a la empresa Sistemas Ancater. Este contrato contenía un convenio arbitral. Posteriormente, las partes celebraron un contrato de mantenimiento para ciertas prestaciones en Ecuador. Este contrato no contenía un convenio arbitral.

La Corte de Apelaciones de París, en una decisión del 21 de junio de 1990, consideró que no era posible la extensión del convenio arbitral del contrato de distribución al contrato de mantenimiento porque no estaban conectados. En particular, la Corte consideró que los contratos de mantenimiento en Ecuador no estaban dentro del contrato de distribución, el mismo que estaba referido exclusivamente a Venezuela, y el contrato de mantenimiento en ninguna parte hacía referencia al contrato de distribución.

\section{B. Cuando existe un convenio arbitral en un contrato y otro contrato del grupo tiene una cláusula judicial}

Los casos descritos versan sobre una situación en la cual, dentro de un grupo de contratos, uno de los contratos tiene una cláusula arbitral y otro de los contratos tiene una cláusula de jurisdicción judicial.

1. Decisión del 29 de noviembre de 1991 de la Corte de Apelaciones de París ${ }^{49}$

En este caso, dos empresas distribuidoras de carros decidieron suscribir un contrato de protocolo que servía como un acuerdo marco. Dos años después, las mismas dos empresas suscribieron contratos de leasing-management, en virtud del cual se comprometieron a unificar su red de distribución en Francia a través de una tercera empresa, lo cual constituía una implementación del contrato de protocolo. Esos contratos contenían una cláusula que remitían las controversias al foro judicial.

Suscitada una controversia entre las partes relacionada a los contratos de leasing-mangagment, se promovió un proceso judicial. La parte demandada solicitó que las controversias de los contratos de leasing-managment debían plantearse en sede arbitral.

La Corte de Apelaciones de París, en sentencia del 29 de noviembre de 1991, determinó que ambos contratos constituían un todo contractual y formaban parte de un mismo grupo de contratos.

Por consiguiente, la Corte consideró que la cláusula arbitral contenida en el acuerdo marco por su redacción amplia también era aplicable a las controversias derivadas de los contratos de implementación, como eran los contratos de leasingmanagement.

En palabras de la Corte de Apelaciones de París:

"In the presence of a protocol constituting the basic agreement between the parties, and leasemanagement contracts signed in performance of this framework agreement and in accordance with the terms and conditions it had laid down, it is worth pointing out that such agreements constitute a contractual whole, a group of contracts, in which the lease-management appears as a means of implementing the protocol. The arbitration clause, which reveals the common will of the parties to the contract, concerns all disputes pertaining thereto and in particular those relative to the consequences of the termination of the leasemanagement contracts concluded in performance of said protocol.

The scope of the arbitration clause as an expression of the will of the parties is far wider than that of a jurisdiction clause, in that it has the effect of vesting the arbitrators with the power to judge, thereby excluding the intervention of State courts, whereas the jurisdiction clause only designates the court which is territorially competent to decide the disputes" ${ }^{\prime 50}$.

Como vemos, la Corte de Apelaciones de París resolvió a favor del arbitraje a pesar de que en los contratos de implementación existía de una cláusula que le otorgaba jurisdicción al Poder Judicial. Sobre este particular, Hanotiau opina que lo decidido por la Corte de Apelaciones de París es criticable. Sostiene que la decisión de la corte hubiera sido correcta si el segundo contrato no tuviera una cláusula de solución de controversias, pero ante la existencia de una cláusula judicial, las cortes debieron decidir que las controversias del segundo contrato debían plantearse en el foro judicial. ${ }^{51}$

Sobre esta misma decisión, Gary Born también comparte la crítica de Hanotiau. En palabras del profesor Gary Born:

49 First Supplement Ch. 1993 Rev. Arb. 617

50 HANOTIAU, Bernard. Óp. cit. p. 138.

51 HANOTIAU, Bernard. Ibid. p. 299. 
"It is debatable whether the court's minimization of the significance of a forum selection clause is universally applicable: in many cases, the contractual choice of particular national courts has substantial commercial and legal importance, and should not necessarily be subjugated to a parallel arbitration agreement" ${ }^{\prime \prime 2}$.

Como vemos, es criticable que se le otorgue más importancia y, por lo tanto, efecto, a una cláusula arbitral por sobre una cláusula de selección de foro en el contexto de un grupo de contratos.

\section{Otros casos judiciales}

Siguiendo el caso anterior, la tendencia de las cortes francesas ha sido la de sostener que debe optarse por el arbitraje si en un grupo de contratos existe una cláusula arbitral en un contrato y, además, existe una cláusula arbitral en otro.

Como revela Gary Born, la Corte de Casación Francesa en sentencia del 17 de marzo de 2010 en el caso Société Komatsu Asia \& Pacitif PTE Ltd v. Société Pac. Auto, determinó que los reclamos cubiertos por una cláusula arbitral y una cláusula de selección de foro debe ser sometidos a arbitraje ${ }^{53}$.

En el mismo sentido, la Corte de Apelaciones de París en sentencia del 11 de abril de 2002 en el caso SA JDA Software France et autres v. SA Kiabi en el contexto de múltiples contratos, determinó que la existencia de una cláusula de solución de controversias judiciales no enerva los efectos a una cláusula arbitral ${ }^{54}$.

Por su parte, el Tribunal Federal Suizo se ha aproximado en sentido contrario. Gary Born explica que el Tribunal Federal Suizo en sentencia del 20 de diciembre de 1995 sostuvo que, ante la falta de evidencia de una intención en sentido contrario, la cláusula de selección de foro contenida en un contrato de transacción reemplazaba el convenio arbitral contenido en el contrato anterior ${ }^{55}$.

\section{Tribunales arbitrales}

Gary Born explica que los tribunales arbitrales han determinado que la inclusión de una cláusula de selección de foro en un contrato y una cláusula arbitral en otro contrato relacionado, es una evidencia que las partes tenían la intención de que las controversias de cada contrato se tramiten en el foro elegido para cada acuerdo. Es decir, no sería posible extender el convenio arbitral de un contrato a otro relacionado.

En palabras de Gary Born:

"many arbitral tribunals appear to have concluded that the inclusion of a forum selection clause in one agreement, and an arbitration clause in a related agreement, will ordinarily signify the parties' expectation for separate dispute resolution mechanisms" ${ }^{\prime 56}$.

En relación a lo anterior es ilustrativo el caso $\mathrm{CCl}$ 4392. En dicho caso, las partes habían celebrado un contrato marco que contenía un convenio arbitral. Adicionalmente, habían celebrado un contrato relacionado a dicho contrato marco. En las condiciones generales de la venta que se encontraban incluidas en el contrato relacionado existía una cláusula que otorgaba jurisdicción a las cortes judiciales. Como reportan Fouchard, Gaillard \& Goldman, en dicho caso el Tribunal Arbitral rechazó extender el convenio arbitral del contrato marco al contrato relacionado, sobre la base de la existencia de una cláusula de foro judicial en el contrato relacionado $^{57}$. Por ello, Fouchard, Gaillard \& Goldman señalan que:

"where a contract containing a clause attributing jurisdiction to the courts is related to another contract containing an arbitration clause, there can be no extension of the arbitration clause to the first contract" ${ }^{\prime 58}$.

En consecuencia, la tendencia de los tribunales arbitrales parece indicar que la existencia de una cláusula de foro judicial en un contrato del grupo no hace posible que se extienda el convenio arbitral de otro contrato del grupo.

\section{Cuando dentro de los múltiples contratos existen convenios arbitrales cuya redacción no es similar}

Como se explicó anteriormente, uno de los elementos básicos a considerar para decidir si se pue-

\footnotetext{
BORN, Gary. Óp cit. p. 1375.

53 BORN, Gary. Ibid. p. 1375.

54 SA JDA Software France et autres v. SA Kiabi. 2003 Rev. Arb. 1252. Sentencia de la Cote de Apelación de París, del 11 Abril de 2002.

55 BORN, Gary. Óp. cit. p. 1375.

56 BORN, Gary. Ibid. p. 1375.

57 FOUCHARD, Philippe; GAILLARD, Emmanuel y GOLDMAN, Berthold. Óp. cit. p. 304.

58 FOUCHARD, Philippe; GAILLARD, Emmanuel y GOLDMAN, Berthold. Ibid. p. 304.
} 
den someter a un solo arbitraje las controversias de varios contratos, es si los convenios arbitrales entre los contratos del grupo son compatibles.

Si estamos frente a un grupo de contratos y todos tienen la misma cláusula arbitral, difícilmente ello generará algún problema que afecte la competencia del tribunal arbitral. Generalmente, las complicaciones se plantean cuando las cláusulas arbitrales son diferentes, al punto de ser incompatibles. Ya se explicó en la sección anterior cuando se está ante una diferencia sustancial existe un problema de compatibilidad que puede afectar que cada controversia se tenga que plantear a través de la cláusula arbitral prevista en su propio contrato.

Justamente esta discusión se planteó en el caso resuelto por la Corte de Apelaciones de París el 11 de abril de 2002. Según relata Hanotiau ${ }^{59}$, las partes habían celebrado un acuerdo marco y tres adendas, en cuya virtud JDA Software y JDA WorIwide otorgaron a Kiabi una licencia para usar un software además de varios servicios relacionados a la implementación del software. El convenio arbitral del acuerdo marco remitía las controversias a un arbitraje ad-hoc bajo las Reglas UNCITRAL, en francés, con un tribunal compuesto por tres árbitros. Resulta que las partes celebraron una cuarta adenda para clarificar las condiciones de la licencia. Esta adenda tenía una cláusula arbitral ad-hoc bajo Reglas UNCITRAL, por un tribunal de 1 o 3 árbitros, con sede París y el procedimiento arbitral debía tramitarse en inglés.

Suscitada una controversia, Kiabi inició un arbitraje. El tribunal arbitral emitió un laudo preliminar de jurisdicción, en el cual determinó que el idioma del arbitraje sería el francés para el acuerdo marco y sus tres primeras adendas e inglés para la cuarta adenda. Además, determinó que las partes estaban autorizadas a emitir sus alegaciones en inglés o en francés.

JDA Software y JDA Worlwide buscaron la anulación del laudo sobre la base de que los árbitros habían excedido los poderes conferidos por las partes al decidir de tal manera el idioma del arbitraje en el laudo parcial. Sin embargo, la Corte de Apelaciones de París determinó que los árbitros no habían excedido los poderes, porque sin perjuicio que el francés era el idioma para el acuerdo marco y las tres primeras adendas y el inglés para la cuarta adenda, las partes podían expresar sus alegacio- nes en cualquiera de los dos idiomas. En suma, la Corte de Apelaciones de París consideró que los convenios arbitrales eran compatibles.

Por su parte, como bien indica Born, cuando los convenios arbitrales son compatibles, las cortes de los Estados Unidos, Francia, Inglaterra, Suiza y Alemania han considerado que un convenio arbitral de un contrato puede extenderse a otro contrato relacionado siempre que los convenios arbitrales sean compatibles ${ }^{60}$. Como vemos, el criterio de compatibilidad es la clave para que se puedan someter las controversias a un mismo arbitraje.

La incorporación de cláusulas arbitrales distintas e incompatibles en diferentes contratos del grupo de contratos, en palabras de Born constituye "strong evidence that disputes under the various agreements were meant to be arbitrated under different dispute resolution provisions - not those of some other contract" ${ }^{\prime 1}$.

Según Born, dicho razonamiento ha sido plasmado en el Laudo de la Cámara de Comercio de Zurich en el caso 273/95 del 31 de mayo de 1996, por la Corte del Octavo Circuito en el caso Nordin v. Nutri/Sys., Inc. y la Corte del Distrito del Sur de Nueva York en el caso Netherlands Curacao Co., NV v. Kenton Corp ${ }^{62}$.

\section{PARTES NO SIGNATARIAS Y MÚLTIPLES CONTRATOS}

En la sección anterior se presentaron algunos casos en los cuales se mezclaban múltiples partes y múltiples contratos. Algunos no revistieron complejidad importante porque los mismos fueron resueltos tratándolos como si se tratara exclusivamente de un problema de múltiples contratos. Ello ocurrió en la decisión de la Corte Suprema de Francia del 30 de marzo de 2004 o en el caso resuelto por la Corte de Apelaciones de París el 11 de abril de 2002. La existencia de múltiples partes no deberá afectar el análisis en un multicontrato.

Dentro del abanico de situaciones complejas que se pueden presentar en un caso concreto, una labor aún más interesante es que en el contexto de un caso de múltiples contratos existan partes no signatarias. Puede ocurrir y, de hecho, sucede que, existan múltiples contratos que no han sido suscritos por las mismas partes.

\footnotetext{
59 HANOTIAU, Bernard. Óp. cit. 292-295.

60 BORN, Gary. Óp. cit. pp. 1371-1372.

61 BORN, Gary. Ibid. p. 1374

62 BORN, Gary. Ibid. p. 1374
} 
Lo anterior sucedió en el conocido caso Kis France v. Societé Générale resuelto por la Corte de Apelaciones de París. ${ }^{63}$ Kis France, una empresa dedicada a la venta de mini-laboratorios para procesar e imprimir fotos, celebró en mayo de 1983 un Basic Agreement con Societé Generale. Este contrato regulaba el esquema de la venta de minilaboratorios en ciertos países a través de leasings en el cual Societe Generale actuaba en su nombre y en representación de sus subsidiarias. A los pocos meses se suscribieron varios contratos locales entre las subsidiarias de Societe Generale y Kis France. En julio de 1983, se suscribió un "Local Agreement" para la venta de mini-laboratorios en Estados Unidos suscrito por Sogelease Corporation (una subsidiaria de Societe Generale) y Kis Corporation (una subsidiaria de Kis France). En la misma fecha se suscribió un "Grenoble Agreement" entre Societe Generale suscrito en su propio nombre y además en representación de Sogelease Corporation y Kis Photo actuando en su propio nombre y además en representación de Kis USA. Finalmente, se suscribió una adenda al Basic Agreement entre Societe Generale en su propio nombre y en representación de Sogelease Corporation y Kis France actuando en su propio nombre y en representación de Kis Corporation.

El Basic Agreement tenía un convenio arbitral que remitía las futuras disputas a un arbitraje administrado bajo los auspicios de la ICC.

Resulta que se generó una controversia derivada del pago de la renta del leasing. Societe Generale y dos de sus subisidiarias (Sogolease Pacifique y Sogolease Corporation) iniciaron un arbitraje contra Kis France y Kis Photo por responsabilidad solidaria de los montos que Kis Corporation supuestamente le debía a Sogolease Corporation bajo el "Basic Agreement", el "Local Agreement" y el "Grenoble Agreement". Ante ello, Kis France y Kis Photo objetaron la competencia del Tribunal Arbitral alegando que Societe Generale no podía reclamar montos adeudados a su subsidiaria estadounidense (Kis Corporation).

En enero de 1989 el Tribunal Arbitral constituido para conocer la controversia determinó que tenía competencia sobre todos los demandantes, sobre todos los demandados y sobre todas las controversias. Luego de ello, los demandados buscaron la anulación del laudo ante la Corte de Apelaciones de París. Dicha Corte confirmó la validez del laudo. En específico, la Corte de Apelaciones de París entendió que los árbitros aplicaron la teoría del grupo de sociedades para extender el convenio arbitral a las partes no signatarias del grupo de sociedades y además que los contratos estaban estrechamente relacionados. En palabras de la Corte de Apelaciones de París:

"In granting the claim filed by Société Générale and its subsidiaries against KIS France and KIS Photo, the arbitrators examined the agreements between the parties and held that the parties' mutual obligations were inexorably linked and that the parent companies played a dominant role vis-à-vis their subsidiaries, which were bound to abide by the former's commercial and financial decisions. KIS France agreed in the Basic Agreement (Art. VII) that it would 'take all necessary measures to ensure that its foreign subsidiaries fulfill their obligations with respect to the local leasing subsidiaries of Société Générale under the present agreement and the Local Agreements to follow'. In the contract of 1 July 1985 it was stipulated that 'the KIS group shall take all necessary measures to ensure that KIS Photo and KIS US fulfill their obligations to the banks under the present agreement' [...]

Consequently, the arbitrators decided the dispute between the parties concerning the scope of KIS France's obligations and correctly applied the arbitration clause - giving it the meaning the parties intended to give it - when considering that the dispute concerned the execution of both the Basic and the Local Agreement. The arbitrators, therefore, respected the arbitration clause which defined their jurisdictional powers [...]"64.

Como vemos, en este caso no solo se extendió el convenio arbitral a partes que no lo suscribieron, sino que además se extendió el convenio arbitral de un contrato a otro que no tenía una cláusula de solución de controversias.

Otro caso emblemático donde coinciden partes no signatarias y múltiples contratos es el Westland ${ }^{65}$. El 29 de abril de 1975, los Emiratos Árabes Unidos, Arabia Saudí, Qatar y Egipto celebraron un acuerdo en el que establecieron la Organización Árabe para la Industrialización (AOI por sus siglas en inglés). El

63 KIS France SA, KIS Photo Industrie SA v. SA Société Générale, Sogelease Pacifique SA y otros. Corte de Apelación [Court of Appeal]. Paris. 31 octubre de1989. En: Albert Jan van den Berg (editor). Yearbook Commercial Arbitration 1991. Volumen 16. Kluwer Law International. 1991. pp. 145-149.

64 KIS France SA, KIS Photo Industrie SA v. SA Société Générale, Sogelease Pacifique SA y otros. Ibid. pp. 4 y 6.

65 Westland Helicopters Ltd. v. Arab Organization for Industrialization y otros. Interim Award. ICC Case 3879. 5 de marzo de 1984. En: Albert Jan van den Berg (editor). Yearbook Commercial Arbitration 1986. Volumen 11. Kluwer Law International. 1986. 
objetivo de este contrato era desarrollar armamento industrial para beneficio de los cuatro Estados.

En enero de 1978, el Alto Comité del AOI celebró un contrato con el Gobierno Británico, en virtud del cual todos los Estados miembros del AOI se comprometieron a garantizar el cumplimiento de las obligaciones que celebraran con empresas británicas.

En febrero de 1978, Westland (un fabricante británico de helicópteros) suscribió con AOI un Shareholders Agrement en el que crearon una empresa denominada Arab British Helicopters Company $(A B H)$, de la cual $A O I$ tenía el $70 \%$ de acciones y Westland las restantes. La finalidad de $\mathrm{ABH}$ era fabricar y vender los helicópteros Lynx que serían fabricados por Westland. El Shareholders Agremeent contenía una cláusula arbitral que sometía a un arbitraje $\mathrm{CCl}$ las controversias derivadas de dicho acuerdo. Ese mismo día, Westland celebró múltiples contratos con $\mathrm{ABH}$.

Luego de que Egipto reconociera al Estado de Israel y que Arabia Saudí, Qatar y Emiratos Árabes Unidos en represalia comunicaran a Egipto su decisión de terminar el AOI, el Estado de Egipto a través de un Decreto Ley disolvió la constitución del AOI. Al haberse disuelto el $\mathrm{AOI}$ y frustrado el $\mathrm{ABH}$, el Shareholders Agreement y los demás contratos suscritos entre Westland y $\mathrm{ABH}$, Westland inició un arbitraje ante la Cámara de Comercio Internacional contra AOI, Emiratos Árabes Unidos, Arabia Saudí, Qatar, Egipto y $\mathrm{ABH}$. AOI. Emiratos Árabes Unidos, Arabia Saudí y Qatar no se apersonaron al arbitraje. Por su parte, $\mathrm{ABH}$ y Egipto objetaron la competencia del tribunal arbitral sobre la base de que no habían suscrito el Shareholders Agreement (que contenía el convenio arbitral) ni ningún otro contrato con Westland. El Tribunal Arbitral determinó que tenía competencia respecto de todos los demandados y todos los contratos, sobre la base del convenio arbitral contenido en el Shareholders Agreement.

En particular, los árbitros concluyeron que:

"the series of documents concluded constitutes an indivisible whole and the four States thus truly demonstrated their desire to act together, by joining together under one name. The similarity of the clauses used in the various contracts can only serve to bear out this interpretation. It follows that the Tribunal is not merely competent as regards each of these States, $A O l a n d A B H$, but is justified in adjudicating upon their cases in one and the same award" ${ }^{\prime 66}$.
Vale la pena mencionar que este caso fue anulado por una corte federal suiza en relación exclusiva a Egipto. Sin embargo, los demás demandados quedaron vinculados por el laudo. Si bien las particularidades propias de este caso son sumamente complejas, nos revelan que en una misma controversia se pueden dar situaciones de partes que no firman el convenio arbitral que está contenido en múltiples contratos y que pueden terminar siendo parte del arbitraje.

\section{CONCLUSIONES}

Conforme los años pasan las transacciones económicas se complejizan cada vez más. Como hemos visto en este artículo, una misma operación económica suele estar compuesta de múltiples partes e implica la celebración de múltiples contratos. Sin embargo, por descuido o falta de conocimiento, las cláusulas arbitrales no suelen estar a la altura de esos contratos complejos.

Gran parte de los problemas discutidos en los casos que hemos mencionado en este artículo pudieron ahorrarse, si las partes hubieran pactado cláusulas arbitrales compatibles. Muchas veces la mejor solución es pactar una cláusula arbitral global. Un pacto claro es, sin duda, el mejor antídoto ante discusiones de cuál fue la intención de las partes, si los contratos se encuentran relacionados o si existe compatibilidad.

En el fondo, recordemos, el arbitraje es hijo del contrato. Por tanto, si las partes desean llevar adelante en un solo arbitraje la discusión de sus controversias nacidas de varios contratos o si, a partir de identificar la relación económica entre ellas, es posible encontrar esa común voluntad, como ha quedado de demostrado de los casos citados, lo que corresponderá será que los árbitros hagan cumplir esa voluntad y que las cortes lo ratifiquen, de ser necesario. .

\section{BIBLIOGRAFÍA}

\section{Legislación}

Reglamento de Arbitraje de la Cámara de Comercio Internacional, 2012.

\section{Doctrina}

BORN, Gary. "International Commercial Arbitration". Segunda Edición. Kluwer Law International. 2014.

66 Westland Helicopters Ltd. v. Arab Organization for Industrialization y otros. Ibídem. 
FOUCHARD, Philippe; GAILLARD, Emmanuel y Berthold GOLDMAN. "Fouchard, Gaillard \& Goldman on International Commercial Arbitration". Kluwer Law International.1999.

HANOTIAU, Bernard. "Complex Arbitrations: Multiparty, Multicontract, Multi-Issue and Class Actions". International Arbitration Law Library. Kluwer Law International. 2006.

LEBOULANGER, Philippe. "Multi-Contract Arbitration". En: Journal of International Arbitration 13. Kluwer Law International. 1996.

MANTILLA SERRANO, Fernando. "Multiple Parties and Multiple Contracts: Divergent or Comparable Issues?" En: Multiparty Arbitration, Dossiers of the ICC Institute of World Business Law 7. Kluwer Law International. 2010.

MARNIX LEIJTEN, Marc Ynzonides y Bommel VAN DER BEND. "A Guide to the NAI Arbitration Rules: Including a Commentary on Dutch Arbitration Law". Kluwer Law International. 2009.

\section{Jurisprudencia}

Agent v. Supplier. Partial Award. ICC (Case 8420. 1996). En: Albert Jan van den Berg (editor), Yearbook Commercial Arbitration 2000. Volumen 15. Kluwer Law International. 2000.

Buyer (Italia) v. Seller (Alemania). Final Award, ICC Case 11440-2003. En: Albert Jan van den Berg (editor). Yearbook Commercial Arbitration 2006. Volumen 31. Kluwer Law International. 2006.

Fletamentos Maritimos SA v. Effjohn International BV (1996. 2 Lloyd's Rep 304 QB). En: “Jurisprudence - England". ASA Bulletin: Association
Suisse de l'Arbitrage. Kluwer Law International 1997. Volume 15. 1997.

France E5. UNI-KOD sarl v. Ouralkali, Corte de Apelación [Court of Appeal]. 31 de mayo de 2001. En: Albert Jan van den Berg (editor). Yearbook Commercial Arbitration 2001. Volumen 26. Kluwer Law International. 2001.

France E6, S.a.r.I. UNI-KOD (Francia) v. Ouralkali (Russian Federation), Corte de Casación [Supreme Court]. (539. F-P). 30 de marzo de 2004. En: Albert Jan van den Berg (editor). Yearbook Commercial Arbitration 2005. Volumen 30. Kluwer Law International 2005.

KIS France SA, KIS Photo Industrie SA v. SA Société Générale, Sogelease Pacifique SA y otros. Corte de Apelación [Court of Appeal]. Paris. 31 octubre de 1989. En: Albert Jan van den Berg (editor). Yearbook Commercial Arbitration 1991. Volumen 16. Kluwer Law International. 1991.

Manufacturer, Subsidiary v. Building supplier. Interim Award. ICC (Case 7929. 1995). En: Albert Jan van den Berg (editor). Yearbook Commercial Arbitration 2000. Volumen 15. Kluwer Law International. 2000.

Société Glencore Grain Rotterdam BV v. Société Afric. Corte de Apelaciones de París. 23 de noviembre de 1999.

SA JDA Software France et autres v. SA Kiabi. 2003 Rev. Arb. 1252. Sentencia de la Cote de Apelación de París, del 11 Abril de 2002.

Westland Helicopters Ltd. v. Arab Organization for Industrialization y otros. Interim Award. ICC Case 3879. 5 de marzo de 1984. En: Albert Jan van den Berg (editor). Yearbook Commercial Arbitration 1986. Volume 11. Kluwer Law International. 1986. 